\title{
Dermatologists as Social Media Contributors During the COVID-19 Pandemic
}

\author{
Shelley K. Uppal, MD; Donovan G. Kearns, BS; Vipawee S. Chat, BS; Jashin J. Wu, MD
}

\section{PRACTICE POINTS}

- With the coronavirus disease 2019 (COVID-19) pandemic, strict physical distancing measures have made patients and providers alike reliant on global digital social networks such as Instagram, Twitter, and Facebook to facilitate information sharing about COVID-19.

- Dermatologists should utilize social media as a platform to post unbiased, evidence-based information regarding manifestations of COVID-19 and guidelines for treatment of skin disease during the global pandemic.

\footnotetext{
(
} n December 31, 2019, cases of a severe pneumonia in patients in Wuhan, Hubei Province, China, were reported to the World Health Organization. ${ }^{1,2}$ The novel coronavirus-severe acute respiratory syndrome coronavirus 2-was identified, and the coronavirus disease 2019 (COVID-19) became a public health emergency of international concern. ${ }^{1}$ In March 2020, the World Health Organization officially characterized COVID-19 as a pandemic. ${ }^{3}$ As of October 2020, more than 42.3 million cases and 1.1 million deaths from COVID-19 have been confirmed worldwide. ${ }^{4}$

As more understanding of severe acute respiratory syndrome coronavirus 2 develops, various cutaneous manifestations of COVID-19 are being uncovered..$^{5}$ The most common cutaneous manifestations of COVID-19 reported in the literature are maculopapular or morbilliform exanthem (36.1\% of cutaneous manifestations), papulovesicular rash $(34.7 \%)$, painful acral red purple papules $(15.3 \%)$, urticaria $(9.7 \%)$, livedo reticularis lesions $(2.8 \%)$, and petechiae $(1.4 \%) .^{5}$

Interestingly, a series of unique cases was identified in April 2020 by a group of dermatologists in Spain. Most patients were children (median age, 13 years) or young adults (median age, 31 years; average age, 36 years; adult age range, $18-91$ years). ${ }^{1}$ Reporting on a representative sample of 6 patients in that series, the group noted that lesions, initially reddish, papular, and resembling chilblains (pernio), progressively became purpuric and flattened in the course of 1 week. Although the lesions presented with some referred discomfort or pain with palpation, they were not highly symptomatic, and no signs of ischemia or Raynaud syndrome were identified. Over time, lesions self-resolved without intervention. Most patients also did not present with what are considered classic COVID-19 signs or symptoms. Only the oldest patient (aged 91 years) presented with a notable respiratory condition; the remaining patients generally were in good health. ${ }^{1}$ Dermatologists in Italy, France, and the United States also have witnessed these COVID-19-associated cutaneous manifestations.

Scientific understanding of COVID-19 and its associated dermatologic symptoms is evolving. Attention has turned to social media to inform and provide possible health solutions during this unprecedented medical crisis. ${ }^{6}$ Strict physical distancing measures have made patients and providers alike reliant on global digital social networks, such as Instagram, Twitter, and Facebook, to facilitate information sharing about COVID-19. ${ }^{7}$ The abundance of nonexpert advice and misinformation on social media makes communication of unbiased expert

Dr. Uppal is from Albany Medical College, New York. Mr. Kearns is from Loma Linda School of Medicine, California. Ms. Chat is from the Medical College of Georgia at Augusta University. Dr. Wu is from the Research and Education Foundation, Irvine, California.

The authors report no conflict of interest.

Correspondence: Jashin J. Wu, MD (jashinwu@gmail.com).

doi:10.12788/cutis.0112 
information difficult. ${ }^{8,9}$ Furthermore, there is a need for dermatologists to provide medical information to patients regarding COVID-19, such as dermatologic manifestations, and clear guidance on immunobiologic or systemic medications during this unprecedented time. ${ }^{9}$

In recent years, dermatologists have established a growing presence on social media, with many recognized as social media influencers with the ability to affect patients' health-related behavior. ${ }^{10}$ Social media frequently has been used by patients to solicit advice regarding skin concerns., ${ }^{9,10}$ Many individuals, in fact, never see a physician after consulting social media for medical concerns or professional advice. ${ }^{9}$

In addition, as of March 2020, more than $61 \%$ of health care workers were found to use social media as a source of COVID-19-related information. ${ }^{11}$ Therefore, dermatologists should utilize social media as a platform to share evidence-based information with the public and other health care workers.

Through social media, dermatologists can post highquality images with clear descriptions to fully characterize skin manifestations in patients with COVID-19. The process of capturing and posting images to the virtual world using a smartphone allows practitioners to gain advice from peers and consultants, share findings with colleagues, and inform the public. ${ }^{12}$ Social media posts of many deidentified clinical images of rashes in COVID-19infected patients already have enabled rapid recognition of skin signs by dermatologists..$^{13}$

Social media sites also are resources where organizations can post updated, evidence-based findings from academic journals. For example, the American Academy of Dermatology and its official journal, the Journal of the American Academy of Dermatology, had more than 22,000 and 27,000 Instagram followers, respectively, as of a March 2020 analysis. ${ }^{14}$ Recent online forums and social media posts contain accessible, graphical, patient-friendly images and information on evidence-based treatments for skin disease during the COVID-19 pandemic. ${ }^{13}$

We should consider initiatives that empower dermatologists to use social media to post unbiased, evidence-based information regarding manifestations of COVID-19 and guidelines for treatment of skin disease during this medical crisis. We hope that dermatologists will help lead the global response to the COVID-19 pandemic and contribute to the evolving knowledge base by characterizing COVID-19-related rashes, understanding their implications, and determining the best evidence for treatment.

\section{REFERENCES}

1. Landa N, Mendieta-Eckert M, Fonda-Pascual P, et al. Chilblain-like lesions on feet and hands during the COVID-19 pandemic. Int $J$ Dermatol. 2020;59:739-743.

2. Phelan AL, Katz R, Gostin LO. The novel coronavirus originating in Wuhan, China: challenges for global health governance. JAMA. 2020;323:709-710.

3. World Health Organization. Coronavirus disease (COVID-19) Situation Report - 133. WHO Website. June 1, 2020. www.who.int/docs /default-source/coronaviruse/situation-reports/20200601-covid-19 -sitrep-133.pdf?sfvrsn=9a56f2ac_4. Accessed October 14, 2020.

4. COVID-19 dashboard by the Center for Systems Science and Engineering (CSSE) at John Hopkins University. John Hopkins Coronavirus Resource Center website. https://coronavirus.jhu.edu /map.html. Accessed October 24, 2020.

5. Sachdeva M, Gianotti R, Shah M, et al. Cutaneous manifestations of COVID-19: report of three cases and a review of literature. J Dermatolog Sci. 2020;98:75-81.

6. Kapoor A, Guha S, Kanti Das M, et al. Digital healthcare: the only solution for better healthcare during COVID-19 pandemic? Indian Heart I. 2020;72:61-64.

7. Limaye RJ, Sauer M, Ali J, et al. Building trust while influencing online COVID-19 content in the social media world. Lancet Digit Health. 2020;2:E277-E278.

8. Chawla S. COVID-19: challenges and opportunities for dermatology response. J Dermatolog Treat. 2020;31:326-326.

9. Schoenberg E, Shalabi D, Wang JV, et al. Public social media consultations for dermatologic conditions: an online survey. Dermatol Online J. 2020;26:6.

10. DeBord LC, Patel V, Braun TL, et al. Social media in dermatology: clinical relevance, academic value, and trends across platforms. J Dermatolog Treat. 2019;30:511-518.

11. Bhagavathula AS, Aldhaleei WA, Rahmani J, et al. Knowledge and perceptions of COVID-19 among health care workers: cross-sectional study. JMIR Public Health Surveill. 2020;6:E19160.

12. Ashique KT, Kaliyadan F, Aurangabadkar SJ. Clinical photography in dermatology using smartphones: an overview. Indian Dermatol Online J. 2015;6:158-163.

13. Madigan LM, Micheletti RG, Shinkai K. How dermatologists can learn and contribute at the leading edge of the COVID-19 global pandemic. JAMA Dermatol. 2020;156:733-734.

14. Guzman AK, Barbieri JS. Response to: "Dermatologists in social media: a study on top influencers, posts, and user engagement" [published online April 20, 2020]. J Am Acad Dermatol. doi:10.1016/j. jaad.2020.03.118 